\title{
Properties of Beryllium-Barium Titanate Dielectrics
}

\author{
Elmer N. Bunting, George R. Shelton, Ansel S. Creamer, and Bernard Jaffe
}

\begin{abstract}
Dielectrics having compositions in the system $\mathrm{BeO}-\mathrm{BaTiO}_{3}-\mathrm{TiO}_{2}$ were matured at $1,240^{\circ}$ to $1,525^{\circ} \mathrm{C}$. Data are given for the compositions, heat-treatments, absorption, and shrinkage. At $25^{\circ} \mathrm{C}$ the dielectric constant $(K)$ and the reciprocal of the power factor $(Q)$ were determined at frequencies of $50,130,1,000$, and 20,000 kilocycles per second and at 3,000 megacycles per second when $K$ is not greater than 50 . At 1 megacycle per second $K$ was determined at temperatures between $-60^{\circ}$ and $+85^{\circ} \mathrm{C}$ and at 130 kilocycles per second, $K$ and $Q$ were determined at temperatures between $25^{\circ}$ and $200^{\circ} \mathrm{C}$. The linear thermal expansion was measured over the temperature range $25^{\circ}$ to $700^{\circ} \mathrm{C}$. It varied from 0.58 to 0.77 percent. The electrical resistivities of a few specimens were measured at $200^{\circ} \mathrm{C}$, using a direct-current potential constantly applied for several days. In some cases the resistivity decreased by a factor of $10^{5}$ over a few days. For specimens of some compositions, $K$ and $Q$ changed with time.
\end{abstract}

\section{Introduction}

Previous studies, by several investigators, of titanate dielectrics have shown that these ceramics are useful in the field of electronic instrumentation [1 to 5]. ${ }^{1}$ Special applications, such as in some miniature electronic devices, necessitate dielectrics in capacitors that will function at elevated temperatures without excessive electrical losses. One of the best insulators at high temperatures is beryllia, and some ceramics containing beryllia are characterized by low electrical losses [6]. Despite these facts and the increased interest in titanate ceramics during the past decade, very little information on beryllia-titania dielectries has been published.

The present paper, dealing with dielectrics composed of beryllia, baria, and titania, is the fourth in a series on ceramic dielectrics made from titania and the oxides of the alkaline earth elements. An extended range in composition for the system BeO$\mathrm{BaO}-\mathrm{TiO}_{2}$ was covered in this investigation in order to reveal dielectrics that may have desirable properties at various temperatures from $-60^{\circ}$ to $+200^{\circ} \mathrm{C}$.

\section{Preparation of Dielectrics and Methods of Test}

Dielectrics having the compositions shown in figure 1 were prepared from reagent quality barium carbonate and beryllia with the commercial grade of titania (grade TMO) used in the preparation of alkaline-earth titanate dielectrics previously investigated $[2,4,5]$.

The methods of preparing these dielectrics and of determining their properties have been previously described $[2,4]$. For measurements of $K$ and $Q$ at $25^{\circ}$ to $200^{\circ} \mathrm{C}$, the silvered test disk rested on a thin flat silver electrode on a hot-plate, and a silver-wire electrode, in a vertical position, touched the top center of the test disk. These silver electrodes were connected to the terminals on the $Q$-meter by short

\footnotetext{
1 Figures in brackets indicate the literature references at the end of this paper
}

lengths (12 in.) of heavy copper wire. Temperatures of the disks on the hot-plate were measured with a calibrated copper-constantan thermocouple (B\&S Gage No. 34) inserted into a small hole extending from the edge to the center of a similar disk placed next to the disk being measured. Due to the small thickness $(0.1$ in.) of the test specimen, the entire piece was maintained within a small temperature gradient. Tests made on specimens of barium titanate by this procedure gave a Curie point between $115^{\circ}$ and $120^{\circ} \mathrm{C}$, indicating an accuracy of about $\pm 3^{\circ} \mathrm{C}$.

For electrical leakage tests on capacitors, matured specimens 0.75 in. square by 0.010 in. thick were coated over an area of $2 \mathrm{~cm}^{2}$ on each face with firedon silver electrodes, and silver lead wires were attached. The specimens were heated in an electric furnace, and the temperature was measured on a mercury-in-glass thermometer placed next to the test piece. Leakage current was measured on a microammeter.

\section{Results and Discussion}

In table 1, data are given for the composition, heat-treatment, absorption, shrinkage, dielectric constant $(K)$, and $Q$-value (reciprocal of the power factor) of mature specimens. The data for a given composition are considered to be the most representative of those obtained from measurements of four to seven specimens.

The effect of composition on the maturing temperature of the specimens is shown in figure 2 , in which the specimens having the same maturing temperature are connected by lines. In all cases, the range in temperature within which mature specimens could be produced was not determined, but this range usually was not more than $25^{\circ} \mathrm{C}$. Of the 33 bodies with ternary compositions, 25 were matured at $1,275^{\circ} \mathrm{C}$, or less, within 1 or 2 hours. Specimens designated BBe63 (table 1) required several heat treatments of 4 - to 6 -hours duration at $1,315^{\circ} \mathrm{C}$ to reach maturity. One or more relatively low quin- 
tuple points in the phase diagram for this system are indicated by the low-maturing temperatures over a wide range in composition.

At $1 \mathrm{Mc} / \mathrm{s}$ and at $25^{\circ} \mathrm{C}$, the variations in values of $K$ and $Q$ with composition are shown in figures 3 and 4 , respectively. These diagrams were constructed from the data on $K$ and $Q$ given in table 1 . In the binary system beryllia-titania, with the substitution of $\mathrm{BeO}$ for $\mathrm{TiO}_{2}$ the values of $K$ decreased from near 100 for $\mathrm{TiO}_{2}$ to 17 for bodies of composition $6 \mathrm{BeO}: \mathrm{TiO}_{2}$. The observed value of $K$ for $\mathrm{BeO}: \mathrm{TiO}_{2}$ was 57 , compared to 71 reported by B. M. Wul [7]. The higher values sometimes found are probably due to the presence of small amounts of the lower oxide of titanium, which has a dielectric constant of several thousand. Q-values remained at several thousand until about 50 weight percent of $\mathrm{BeO}$ was present. Higher percentages of $\mathrm{BeO}$ resulted in specimens with lower $Q$, which decreased to 130 for $6 \mathrm{BeO}: \mathrm{TiO}_{2}$. The dielectric constant and $Q$-values for $\mathrm{BeO}\left(99.7 \%\right.$ pure), matured at $1,925^{\circ} \mathrm{C}$ and measured at $25^{\circ} \mathrm{C}$ and $100 \mathrm{kc} / \mathrm{s}$, have been reported as $K=6.3$ and $Q=420$ [6].

Within the ternary system, for dielectrics containing percentages of $\mathrm{TiO}_{2}$ greater than about 60 , the replacement of $\mathrm{BaO}$ by $\mathrm{BeO}$ did not change the values of $K$ very much, but usually increased the $Q$-values. However, on the join $3 \mathrm{BeO}: \mathrm{TiO}_{2}$ $\mathrm{BaO}: 2 \mathrm{TiO}_{2}, K$ decreased from 200 for $\mathrm{BaO}: 2 \mathrm{TiO}_{2}$ to 30 for $3 \mathrm{BeO}: \mathrm{TiO}_{2}$, with a minimum of 25 about midway along the join. The $Q$-values usually increased as the $\mathrm{BeO}$ content increased. On the $4 \mathrm{BeO}: \mathrm{TiO}_{2}-2 \mathrm{BaO}: 3 \mathrm{TiO}_{2}$ join, $K$ decreased rapidly from 900 for $2 \mathrm{BaO}: 3 \mathrm{TiO}_{2}$ to 23 for $4 \mathrm{BeO}: \mathrm{TiO}_{2}$. The addition of 9 weight percent of $4 \mathrm{BeO}: \mathrm{TiO}_{2}$ to $2 \mathrm{BaO}: 3 \mathrm{TiO}_{2}$ decreased $Q$ from 50 for $2 \mathrm{BaO}: 3 \mathrm{TiO}_{2}$ to 33 for $2 \mathrm{BBe} 49$ (table 1 ), but greater percentages of $4 \mathrm{BeO}: \mathrm{TiO}_{2}$ increased the $Q$ to a maximum of 3,000 at about 90 percent. With more than 90 percent, a decrease in $Q$ to 300 for $4 \mathrm{BeO}: \mathrm{TiO}_{2}$ was found. On the $6 \mathrm{BeO}: \mathrm{TiO}_{2}-\mathrm{BaTiO}_{3}$ join, $K$ decreased from about 1,500 for $\mathrm{BaTiO}_{3}$ to 17 for $6 \mathrm{BeO}: \mathrm{TiO}_{2}$. Q-values decreased from about 100 for $\mathrm{BaTiO}_{3}$ to 30 when the content of $6 \mathrm{BeO}: \mathrm{TiO}_{2}$ was 25 percent (BBe67, table 1). Further additions of $6 \mathrm{BeO}: \mathrm{TiO}_{2}$, increased the $Q$ to a maximum of 8,000 at 90 percent. However, at 100 percent of $6 \mathrm{BeO}: \mathrm{TiO}_{2}$, the $Q$ had decreased to 130 .

The effect of variation in frequency on the $Q$-values may also be noted in table 1 . In the large majority of specimens, the $Q$-values were lower at $50 \mathrm{kc} / \mathrm{s}$ than at the higher frequencies. At $20 \mathrm{Mc} / \mathrm{s}$, they were usually higher than at $1 \mathrm{Mc} / \mathrm{s}$ for specimens of high titania content, but lower for those of high beryllia or barium titanate content. At 3,000 Mc/s, higher values of $Q$ were found than at $20 \mathrm{Mc} / \mathrm{s}$ for about half of the specimens tested.

In some miniaturized electronic equipment, the parts are subjected to temperatures of about $200^{\circ} \mathrm{C}$, necessitating a knowledge of the properties of the dielectrics under these conditions. Data are given, in table 2 , for $K$ and $Q$ measured at $130 \mathrm{kc} / \mathrm{s}$ at temperatures between $25^{\circ}$ and $200^{\circ} \mathrm{C}$, and for the average temperature coefficient of $K$. These data, in most instances, were obtained on one specimen only of each composition. For most of these dielectrics, $K$ decreased with increasing temperature, and the average temperature coefficient of $K$ ranged from -35 to $-810 \mathrm{ppm} /{ }^{\circ} \mathrm{C}$. $Q$-values usually were decreased greatly at higher temperatures, and at $200^{\circ} \mathrm{C}$ only seven specimens had $Q$ greater than 500 , with the highest $(1,400)$ for $2 \mathrm{BBe} 8$. Only 10 of the 54 test pieces had a higher $G$ at $200^{\circ} \mathrm{C}$ than at $25^{\circ} \mathrm{C}$, and most of the 10 were of high barium titanate content. The "Q" meter was warm and under voltage control of $\pm 1 / 2$ percent when the measurements were made on specimens having a capacitance of 100 to $200 \mu \mu \mathrm{f}$. Values of the average temperature coefficient of $K$ are probably within $\pm 20 \mathrm{ppm} /{ }^{\circ} \mathrm{C}$. The temperature of the test pieces was raised at the rate of about $3 \mathrm{deg} \mathrm{C}$ a minute, and readings were taken at 25 deg C intervals. In order to illustrate the variations in $Q$ resulting from changes in temperature and composition, figures 5 and 6 were constructed for temperatures of $100^{\circ}$ and $200^{\circ} \mathrm{C}$, respectively, and are based on the data in table 2 .

In table 3 data are given for the values of $K$, at $1 \mathrm{Mc} / \mathrm{s}$, over the temperature range of $-60^{\circ}$ to $+85^{\circ} \mathrm{C}$ and for the average temperature coefficient of $K$. These values were obtained by measuring the capacitance of test disks at $10-\mathrm{deg}$ intervals, with the temperature held constant at each interval for at least $15 \mathrm{~min}$ before measurements were made. The average values of the temperature coefficient of $K$, last column of table 3 , are considered to be not better than $\pm 10 \mathrm{ppm} /{ }^{\circ} \mathrm{C}$, and most of them are negative within the range -40 to $-840 \mathrm{ppm} /{ }^{\circ} \mathrm{C}$. In order to illustrate the variation of $K$ resulting from changes in temperature and composition, figures 7,8 , and 9 were constructed for temperatures of $-60^{\circ},+100^{\circ}$, and $+200^{\circ} \mathrm{C}$, respectively. These figures contain isodielectric-constant lines, based on the data in tables 2 and 3.

After storage for 6 months under room conditions, two or more specimens of each composition were retested for $K$ and $Q$ at $25^{\circ} \mathrm{C}$ and $1 \mathrm{Mc} / \mathrm{s}$. The results for the dielectrics that showed a significant change are given in table 4 . These changes, particularly in $K$, are not as large, on the average, as those found to occur in the titanate systems previously studied $[2,4,5]$. Specimens of only one composition (BBe69), of high $\mathrm{BaTiO}_{3}$ content, decreased in $K$ and at the same time increased in $Q$ by an appreciable amount. The absence of much solid solution between $\mathrm{BeO}$ and $\mathrm{BaTiO}_{3}$ may account for the smaller change, with time, in the electrical properties of these dielectrics compared to those containing the other alkaline-earth oxides.

Linear thermal expansions, determined by the interferometer method, were moderately high for representative specimens, as shown in table 5 .

In order to determine the usefulness of these dielectrics in capacitors at $200^{\circ} \mathrm{C}$ and under a constant potential, tests of the change in electrical resistivity over a period of time were made on some specimens of various compositions. On the assumption that practically all of the conductivity was 
through the piece, the approximate volume resistivity was calculated from the amount of current and the dimensions of the test piece between the fired-on silver electrodes. The data obtained are shown in table 6 and figures 10 and 11 . Barium titanate specimens showed the fastest and largest decrease in resistivity, while the wafers of high beryllia content showed the least change in resistance. After the tests, only those specimens exhibiting a considerable decrease in resistivity were found to be colored black throughout the volume between the silver electrodes, while portions not under the electrodes remained unchanged in color. This decrease in resistivity and change in color is probably due to the reduction of the tetravalent titanium to the trivalent form. A reversal in the polarity of the applied voltage resulted in a temporary increase in resistivity, indicating a partial reoxidation. The most variable resistivity data were obtained for specimens of barium titanate. One of the factors most likely to influence the resistivity is the number of dark-colored spots in the specimen before the test. The data on barium titanate given in table 6 and figure 10 are the best values for specimens made with a commercial grade of titania. At $200^{\circ} \mathrm{C}$ and under $3-\mathrm{v}$ direct current per mil, the resistivity for $\mathrm{BaTiO}_{3}$ is $1.0 \times 10^{8} \mathrm{ohm}-$ $\mathrm{cm}$ when measured immediately after applying the voltage. For the other specimens given in table 6 , the resistivity just after application of the voltage is greater than $10^{10} \mathrm{ohm}-\mathrm{cm}$. In order to find how much voltage was needed to cause a decrease in resistivity with time at $200^{\circ} \mathrm{C}$, the voltage on specimen BBe67 was increased to $100(10 \mathrm{v} / \mathrm{mil})$ after the specimen had been at $30 \mathrm{v}$, and $200^{\circ} \mathrm{C}$, for 21 days. The resistivity remained greater than $10^{10} \mathrm{ohm}-\mathrm{cm}$ for 4 additional days and then decreased to $2 \times 10^{7} \mathrm{ohm}-\mathrm{cm}$.

The decrease in resistivity shown by these dielectrics, when subjected to a constant voltage, greatly lowers their usefulness for high-temperature duty. Those bodies, however, which showed a small loss in resistivity at $200^{\circ} \mathrm{C}$ could be used for the production of capacitors for operation at this temperature if the working voltage is not too high.

\section{Summary}

Dielectrics having compositions in the system $\mathrm{BeO}-\mathrm{BaTiO}_{3}-\mathrm{TiO}_{2}$ can be prepared from mixtures of titania with barium carbonate and beryllia. Mature dielectrics (less than $0.1 \%$ absorption) were made by heating specimens to various temperatures within the range $1,240^{\circ}$ to $1,525^{\circ} \mathrm{C}$.
The dielectric constant $(K)$ varied from 16 for specimens with compositions near that of $6 \mathrm{BeO}$ :$\mathrm{TiO}_{2}$, to several hundred for dielectrics of high baria content. Most of the temperature coefficients of $K$ were negative. The $Q$-values, at $25^{\circ} \mathrm{C}$, ranged from 15 to 10,000 and were low for dielectrics containing large percentages of baria and for those with high beryllia content in the binary system $\mathrm{BeO}_{-} \mathrm{TiO}_{2}$. At higher temperatures, most of the $Q$-values decreased greatly. $K$ and $Q$-values of some of the dielectrics changed with time,

Values for the linear thermal expansion over the temperature range $25^{\circ}$ to $700^{\circ} \mathrm{C}$, varied from 0.58 to 0.77 percent and were obtained on dielectrics of widely different compositions.

When subjected to an electrical potential at $200^{\circ} \mathrm{C}$ for many hours, some of the dielectrics decreased greatly in electrical resistivity.

\section{References}

[1] A. von Hippel et al., Ind. Eng. Chem. 38, 1097 (1946).

[2] E. N. Bunting, G. R. Shelton, and A. S. Creamer, J. Research NBS 38, 337 (1947) RP1776; J. Am. Ceram. Soc. 30, 4 (1947).

[3] U. S. Pat. 2,420,692, by E. Wainer.

[4] G. R. Shelton, A. S. Creamer, and E. N. Bunting, J. Research NBS 41, 17 (1948) RP1899.

[5] E. N. Bunting, G. R. Shelton, and A. S. Creamer, J. Research NBS 43, 237 (1949) RP2025.

[6] R. F. Geller, P. J. Yavorsky, B. L. Steirman, and A. S. Creamer, J. Research NBS 36, 277 (1946) RP1703.

[7] B. M. Wul, Nature 156, $480(1945)$.

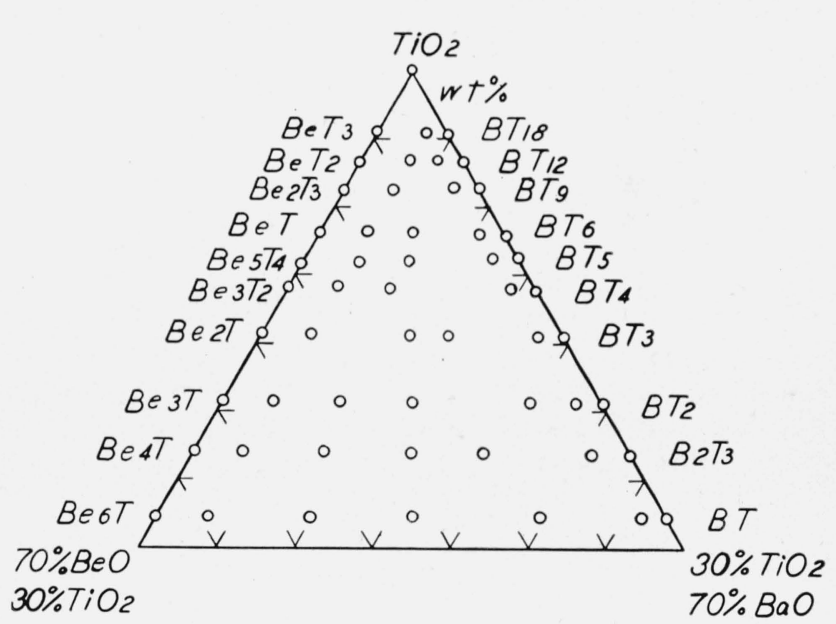

Figure 1. Ternary diagram for system $\mathrm{BeO}-\mathrm{BaTiO}_{3}-\mathrm{TiO}_{2}$ showing compositions studied.

$\mathrm{B}=\mathrm{BaO} ; \mathrm{Be}=\mathrm{BeO} ; \mathrm{T}=\mathrm{TiO}_{2} ;$ thus $\mathrm{Be} 6 \mathrm{~T}=6 \mathrm{BeO}: \mathrm{TiO}_{2}$. 


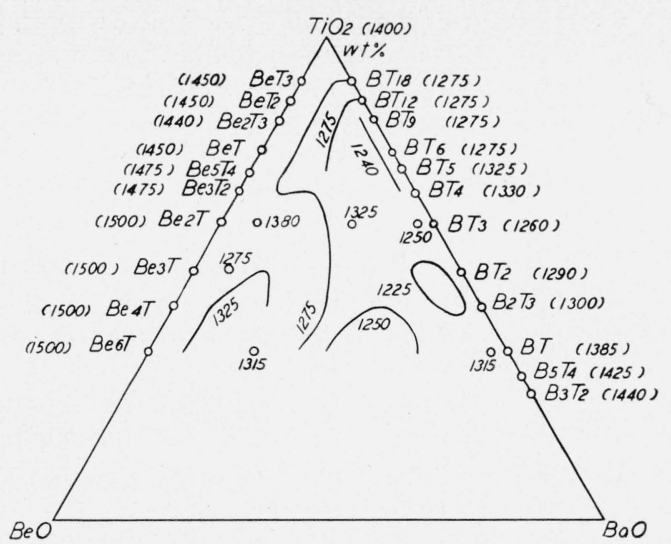

Figure 2. Approximate maturing temperature $\left({ }^{\circ} \mathrm{C}\right)$ after calcining treatment.

$\mathrm{B}=\mathrm{BaO} ; \mathrm{Be}=\mathrm{BeO} ; \mathrm{T}=\mathrm{TiO}_{2}$

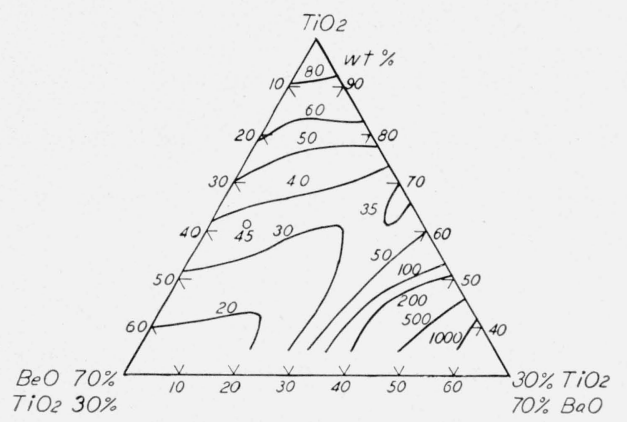

Figure 3. Dielectric constant, at $25^{\circ} \mathrm{C}$ and $1 \mathrm{Mc} / \mathrm{s}$, with varying composition within the ternary system $\mathrm{BeO}-\mathrm{BaTiO}_{3}-$ $\mathrm{TiO}_{2}$.

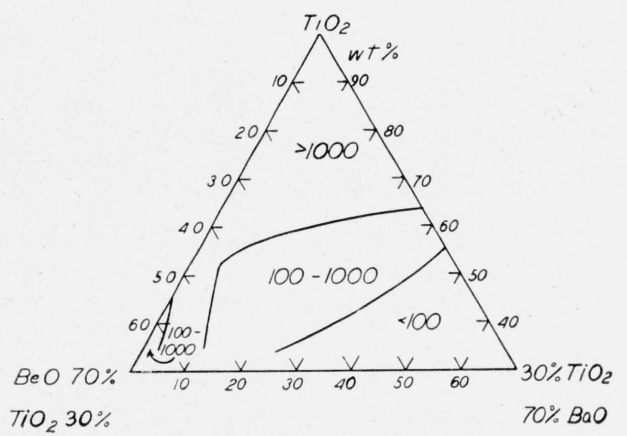

FIGURE 4. Q-values, at $25^{\circ} \mathrm{C}$ and $1 \mathrm{Mc} / \mathrm{s}$, with varying composition within the ternary system $\mathrm{BeO}-\mathrm{BaTiO}_{3}-\mathrm{TiO}_{2}$.

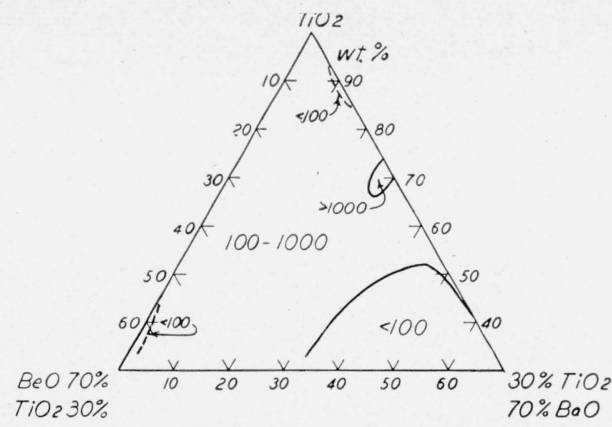

Figure 5. Q-values, at $100^{\circ} \mathrm{C}$ and $130 \mathrm{kc} / \mathrm{s}$, with varying composition within the ternary system $\mathrm{BeO}-\mathrm{BaTiO}_{3}-\mathrm{TiO}_{2}$.

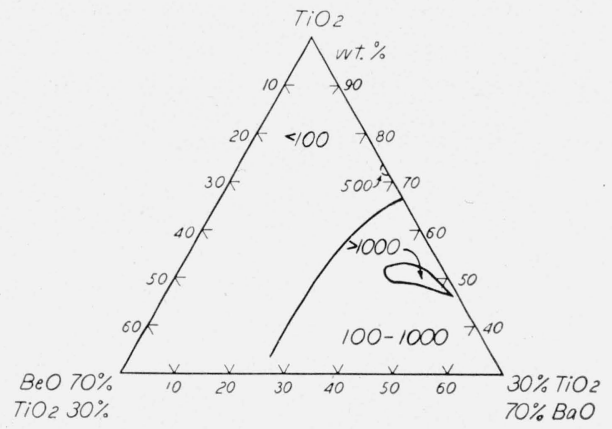

FIgure 6. Q-malues, at $200^{\circ} \mathrm{C}$ and $130 \mathrm{kc} / \mathrm{s}$, with varying composition within the ternary system $\mathrm{BeO}-\mathrm{BaTiO}_{3}-\mathrm{TiO}_{2}$.

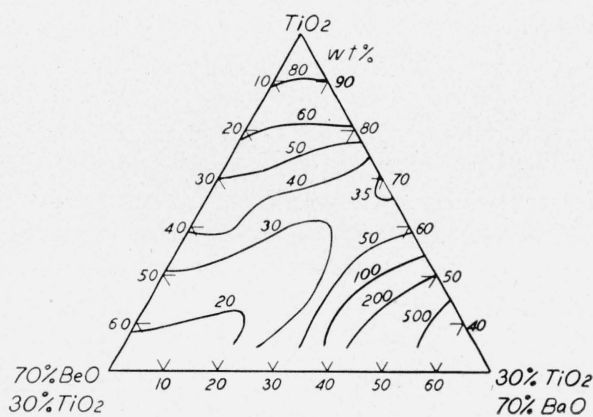

Figure 7. Dielectric constant, at $-60^{\circ} \mathrm{C}$ and $1 \mathrm{Mc} / \mathrm{s}$, with varying compositıon within the ternary system $\mathrm{BeO}-\mathrm{BaTiO}_{3}-$ $\mathrm{TiO}_{2}$. 


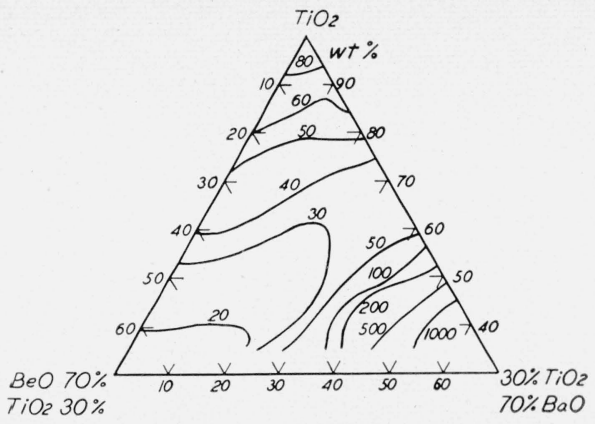

Figure 8. Dielectric constant, at $100^{\circ} \mathrm{C}$ and $130 \mathrm{kc} / \mathrm{s}$, with varying composition within the ternary system $m_{2} \mathrm{BeO}-\mathrm{BaTiO}_{3-}$ $\mathrm{TiO}_{2}$.

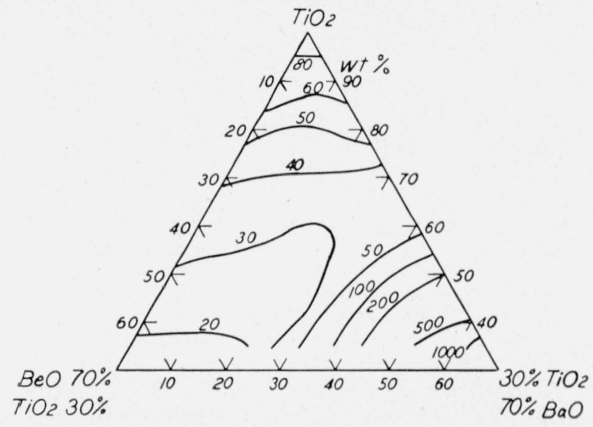

Figure 9. Dielectric constant, at $200^{\circ} \mathrm{C}$ and $130 \mathrm{kc} / \mathrm{s}$, with varying composition within the ternary system $\mathrm{BeO}-\mathrm{BaTiO}_{3}-$ $\mathrm{TiO}_{2}$.

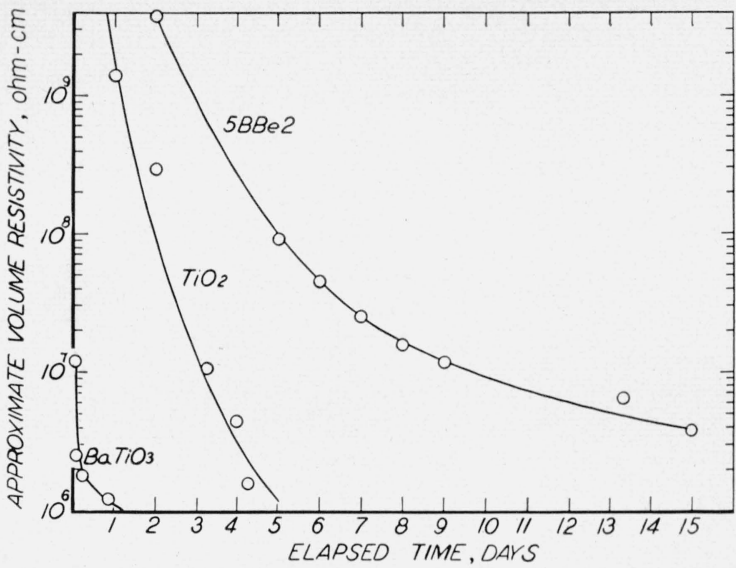

FIGURE 10. Approximate volume resistivity for specimens of $\mathrm{BaTiO}_{3}, \mathrm{TiO}_{2}$, and $5 \mathrm{BBe}_{2}$, at $200^{\circ} \mathrm{C}$ and 30 volts directcurrent, after varying lengths of time.

Thickness of all specimens was approximately $0.010 \mathrm{in}$

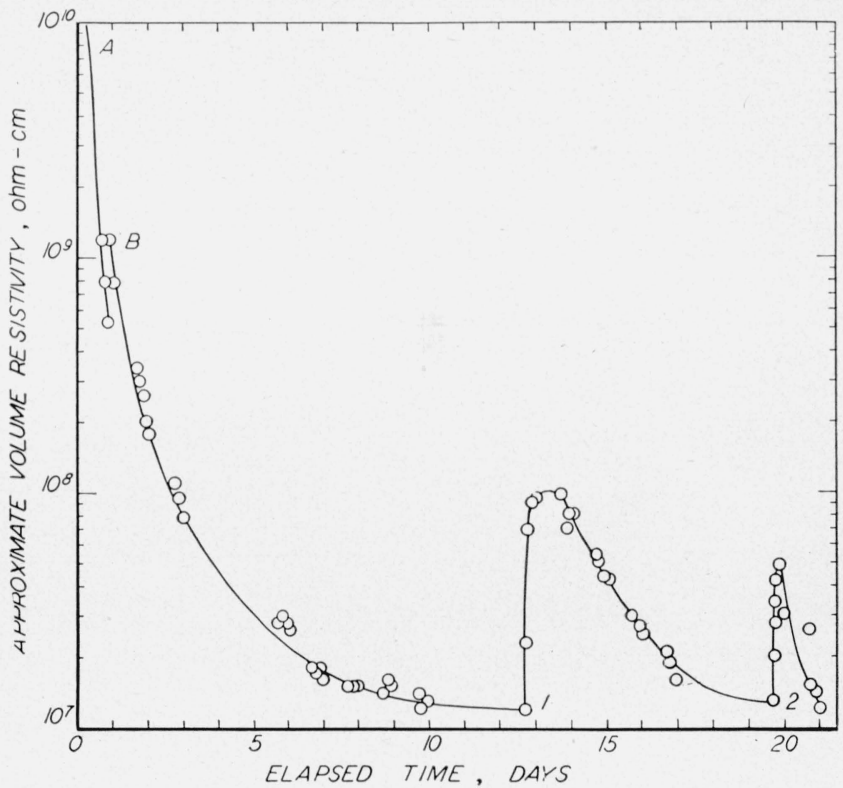

FIGURE 11. Approximate volume resistivity for specimens of $5 \mathrm{BBe}_{2}, 0.010$ in. thick, at $200^{\circ} \mathrm{C}$ and 110 or 30 volts directcurrent, after varying lengths of time.

A, $110 \mathrm{v} ; \mathrm{B}, 30 \mathrm{v} ; 1$, polarity reversed; 2 , original polarity restored. 
TABLE 1. Composition, heat-treatment, absorption, shrinkage, dielectric constant $K$, and $Q$ of bodies in the system 6 $\mathrm{BeO}: \mathrm{TiO}_{2}-\mathrm{BaTiO}_{3}-\mathrm{TiO}_{2}$

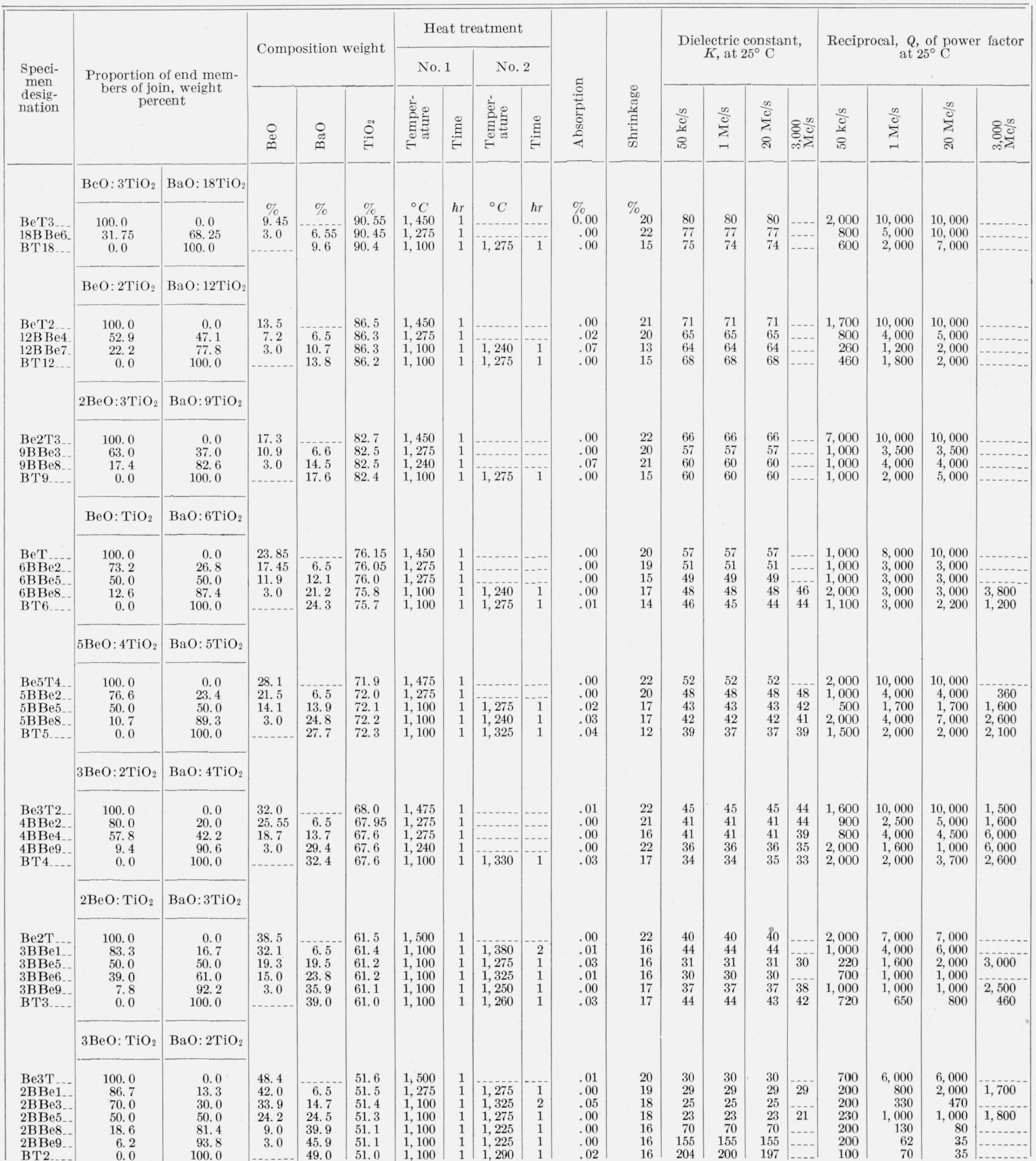


TABLE 1. Composition, heat-treatment, absorption, shrinkage, dielectric constant $K$, and $Q$ of bodies in the system 6 $\mathrm{BeO}: \mathrm{TiO}_{2}-\mathrm{BaTiO}_{3}-\mathrm{TiO}_{2}-$ Continued.

\begin{tabular}{|c|c|c|c|c|c|c|c|c|c|c|c|c|c|c|c|c|c|c|c|}
\hline \multirow{3}{*}{$\begin{array}{l}\text { Speci- } \\
\text { men } \\
\text { desig- } \\
\text { nation }\end{array}$} & \multirow{3}{*}{\multicolumn{2}{|c|}{$\begin{array}{l}\text { Proportion of end mem- } \\
\text { bers of join, weight } \\
\text { percent }\end{array}$}} & \multirow{2}{*}{\multicolumn{3}{|c|}{ Composition weight }} & \multicolumn{4}{|c|}{ Heat treatment } & \multirow[b]{3}{*}{$\begin{array}{l}\tilde{0} \\
\stackrel{0}{0} \\
0 \\
0 \\
0 \\
0 \\
0\end{array}$} & \multirow[b]{3}{*}{ 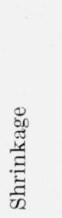 } & \multirow{2}{*}{\multicolumn{4}{|c|}{$\begin{array}{c}\text { Dielectric constant, } \\
K \text {, at } 25^{\circ} \mathrm{C}\end{array}$}} & \multirow{2}{*}{\multicolumn{4}{|c|}{$\begin{array}{c}\text { Reciprocal, } Q \text {, of power factor } \\
\text { at } 25^{\circ} \mathrm{C}\end{array}$}} \\
\hline & & & & & & \multicolumn{2}{|c|}{ No. 1} & \multicolumn{2}{|c|}{ No. 2} & & & & & & & & & & \\
\hline & & & $\underset{\infty}{\infty}$ & $\underset{\nexists ָ}{\stackrel{0}{\sim}}$ & $\stackrel{0}{E}$ & 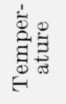 & $\stackrel{\mathscr{\Xi}}{E}$ & 离兽 & 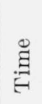 & & & $\frac{n}{0}$ & $\sum_{-1}^{\infty}$ & $\frac{\sum^{n}}{0}$ & $\underset{\infty}{\oint_{\infty}} \frac{\infty}{0}$ & 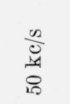 & $\sum_{-1}^{\infty}$ & 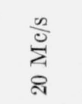 & $\sum_{\infty}^{\infty} \frac{\pi}{0}$ \\
\hline & $4 \mathrm{BeO}: \mathrm{TiO}_{2}$ & $2 \mathrm{BaO}: 3 \mathrm{TiO}_{2}$ & & & & & & & & & & & & & & & & & \\
\hline Be4T. & 100.0 & 0.0 & $\begin{array}{r}\% \\
55.6\end{array}$ & $\%$ & $\begin{array}{r}\% \\
44.4\end{array}$ & $\begin{array}{c}{ }^{\circ} \mathrm{C} \\
1,500\end{array}$ & $\begin{array}{c}h r \\
1\end{array}$ & ${ }^{\circ} \mathrm{C}$ & $h r$ & $\begin{array}{l}\% \\
.00\end{array}$ & ${ }^{\%}{ }_{21}$ & 23 & 23 & 23 & & 90 & 300 & 440 & \\
\hline 2BBe41 & 88.4 & 11.6 & 49.2 & 6.5 & 44.3 & 1,100 & 1 & 1,325 & 1 & .00 & 18 & 23 & 23 & 23 & 23 & 900 & 3,000 & 3,000 & 2,000 \\
\hline $2 \mathrm{BBe} 43$ & 70.0 & 30.0 & 38.9 & 16.8 & 44. 2 & 1,100 & 1 & 1,325 & 3 & .01 & 16 & 21 & 21 & 21 & & 300 & 600 & 1,000 & \\
\hline $2 \mathrm{BBe} 45$ & 50.0 & 50.0 & 27.8 & 28.1 & 44.1 & 1,100 & 1 & 1,275 & 1 & .01 & 16 & 27 & 27 & 27 & 24 & 300 & 270 & 190 & 73 \\
\hline $2 \mathrm{BBe} 47$ & 25.0 & 75.0 & 13.9 & 42.1 & 44. 0 & 1,100 & 1 & 1,250 & 2 & .01 & 16 & 170 & 165 & 155 & -... & 53 & 36 & 20 & \\
\hline $2 \mathrm{BBe} 49$ & 9.0 & 91.0 & 5.0 & 51.1 & 43.9 & 1,100 & 1 & 1,225 & 1 & .00 & 16 & 490 & 470 & 450 & ... & 45 & 33 & 18 & \\
\hline B2T3 & 0.0 & 100.0 & ....... & 56.1 & 43.9 & 1,250 & 1 & 1,300 & 1 & .01 & 11 & 910 & 900 & 890 & $\ldots$ & 70 & 50 & 25 & \\
\hline & $6 \mathrm{BeO}: \mathrm{TiO}_{2}$ & $\mathrm{BaO}: \mathrm{TiO}_{2}$ & & & & & & & & & & & & & & & & & \\
\hline Be6T & 100.0 & 0.0 & 65.2 & & 34.8 & 1,525 & 1 & & & .07 & 21 & 17 & 17 & 17 & $\ldots$ & 40 & 130 & 110 & \\
\hline BBe61.- & 90.1 & 9.9 & 58.8 & 6.5 & 34.7 & 1,100 & 1 & 1,325 & 3 & .08 & 18 & 16 & 16 & 16 & & 800 & 8,000 & 10,000 & \\
\hline BBe63.- & 70.0 & 30.0 & 45.7 & 19. 7 & 34.6 & 1,100 & 1 & 1,315 & 14 & .08 & 15 & 17 & 17 & 17 & 15 & 110 & 130 & 600 & 950 \\
\hline BBe65 & 50.0 & 50.0 & 32.6 & 32.9 & 34.5 & 1,100 & 1 & 1,250 & 1 & .00 & 18 & 65 & 64 & 61 & .... & 91 & 68 & 40 & \\
\hline ВBe67.- & 25.0 & 75.0 & 16.3 & 49.3 & 34.4 & 1,100 & 1 & 1,250 & 2 & .02 & 14 & 680 & 660 & 620 & $\ldots$ & 49 & 30 & 15 & $\ldots$ \\
\hline ВBe69 & 4. 6 & 95.4 & 3.0 & 62.7 & 34.3 & 1,100 & 1 & 1,325 & 2 & .00 & 14 & 1,170 & 1,140 & 1,120 & $\ldots$ & 86 & 60 & 26 & \\
\hline BT & 0.0 & 100.0 & ...... & 65.7 & 34.3 & 1,245 & 1 & 1,385 & 2 & .05 & 11 & 1,500 & 1,500 & 1,500 & & 80 & 100 & 50 & \\
\hline
\end{tabular}


TABLE 2. Dielectric constant $(K), Q$-values, and average temperature coefficient of $K$ from $25^{\circ}$ to $200^{\circ} \mathrm{C}$, at $130 \mathrm{kc} / \mathrm{s}$

\begin{tabular}{|c|c|c|c|c|c|c|c|c|c|c|c|c|c|}
\hline \multirow{2}{*}{$\begin{array}{c}\text { Specimen designa- } \\
\text { tion }\end{array}$} & \multicolumn{6}{|c|}{$K$ at ${ }^{\circ} \mathrm{C}-$} & \multicolumn{6}{|c|}{$Q$ at ${ }^{\circ} \mathrm{C}-$} & \multirow{2}{*}{$\begin{array}{l}\text { Average } \\
\text { temperature } \\
\text { coefficient } \\
\text { of } K\end{array}$} \\
\hline & $25^{\circ}$ & $100^{\circ}$ & $125^{\circ}$ & $150^{\circ}$ & $175^{\circ}$ & $200^{\circ}$ & $25^{\circ}$ & $100^{\circ}$ & $125^{\circ}$ & $150^{\circ}$ & $175^{\circ}$ & $200^{\circ}$ & \\
\hline $\mathrm{TiO}_{2} \ldots$ & 98 & 92 & 90 & 89 & 88 & 86 & 3,000 & 670 & 230 & 140 & 112 & 43 & $\begin{array}{r}p p m /{ }^{\circ} C \\
-770\end{array}$ \\
\hline $\begin{array}{l}\text { BeT3 } \\
\text { 18BBe6.. } \\
\text { BT18 }\end{array}$ & $\begin{array}{l}78.2 \\
77.8 \\
75.2\end{array}$ & $\begin{array}{l}74.8 \\
73.9 \\
71.7\end{array}$ & $\begin{array}{l}73.4 \\
73.1 \\
71.0\end{array}$ & $\begin{array}{l}72.4 \\
72.2 \\
70.6\end{array}$ & $\begin{array}{l}71.1 \\
71.9 \\
69.2\end{array}$ & $\begin{array}{r}70.2 \\
\hdashline-.-\end{array}$ & $\begin{array}{r}4,000 \\
1,200 \\
560\end{array}$ & $\begin{array}{r}670 \\
190 \\
46\end{array}$ & $\begin{array}{r}420 \\
100 \\
26\end{array}$ & $\begin{array}{r}230 \\
46 \\
14\end{array}$ & $\begin{array}{r}91 \\
20 \\
8\end{array}$ & 48 & $\begin{array}{l}-700 \\
-525 \\
-550\end{array}$ \\
\hline $\begin{array}{l}\text { BeT2 } \\
\text { 12BBe4 } \\
\text { 12BBe7 } \\
\text { BT12 }\end{array}$ & $\begin{array}{l}68.8 \\
65.9 \\
59.1 \\
69.6\end{array}$ & $\begin{array}{l}64.7 \\
62.6 \\
56.7 \\
66.8\end{array}$ & $\begin{array}{l}63.5 \\
61.7 \\
56.0 \\
66.3\end{array}$ & $\begin{array}{l}62.4 \\
61.0 \\
55.6 \\
66.0\end{array}$ & $\begin{array}{r}61.6 \\
55.3 \\
-\end{array}$ & \begin{tabular}{r}
60.7 \\
\hdashline-1 \\
$-\ldots$.
\end{tabular} & $\begin{array}{r}4,000 \\
1,400 \\
300 \\
850\end{array}$ & $\begin{array}{r}630 \\
160 \\
240 \\
59\end{array}$ & $\begin{array}{r}370 \\
69 \\
100 \\
31\end{array}$ & $\begin{array}{r}160 \\
30 \\
52 \\
19\end{array}$ & $\begin{array}{r}84 \\
23 \\
-2\end{array}$ & \begin{tabular}{r}
38 \\
\hdashline-1 \\
\end{tabular} & $\begin{array}{l}-720 \\
-620 \\
-440 \\
-420\end{array}$ \\
\hline $\begin{array}{l}\text { Be2T3 } \\
\text { 9BBe3 } \\
9 \mathrm{BBe} 8 \\
\text { BT9..... }\end{array}$ & $\begin{array}{l}66.4 \\
57.8 \\
61.2 \\
57.4\end{array}$ & $\begin{array}{l}62.5 \\
54.8 \\
58.8 \\
55.4\end{array}$ & $\begin{array}{l}61.3 \\
54.0 \\
58.2 \\
54.8\end{array}$ & $\begin{array}{l}60.4 \\
53.5 \\
57.7 \\
54.4\end{array}$ & $\begin{array}{r}59.8 \\
54.2\end{array}$ & 54.2 & $\begin{array}{r}4,000 \\
1,100 \\
460 \\
2,000\end{array}$ & $\begin{array}{l}340 \\
200 \\
140 \\
210\end{array}$ & $\begin{array}{r}120 \\
80 \\
57 \\
89\end{array}$ & $\begin{array}{l}46 \\
34 \\
25 \\
48\end{array}$ & $\begin{array}{r}21 \\
27\end{array}$ & 16 & $\begin{array}{l}-700 \\
-610 \\
-460 \\
-340\end{array}$ \\
\hline $\begin{array}{l}\text { BeT } \\
6 \mathrm{BBe} 2 \\
6 \mathrm{BBe} 5 \\
6 \mathrm{BBe} 8 \\
\text { BT6 }\end{array}$ & $\begin{array}{l}54.2 \\
50.8 \\
49.5 \\
48.5 \\
48.8\end{array}$ & $\begin{array}{l}51.3 \\
48.6 \\
47.7 \\
47.3 \\
48.0\end{array}$ & $\begin{array}{l}50.6 \\
48.1 \\
47.2 \\
47.0 \\
47.9\end{array}$ & $\begin{array}{l}49.9 \\
47.6 \\
46.9 \\
46.7 \\
47.8\end{array}$ & $\begin{array}{l}49.4 \\
47.4 \\
46.5 \\
46.6 \\
47.8\end{array}$ & $\begin{array}{r} \\
\cdots \\
\cdots\end{array}$ & $\begin{array}{r}2,000 \\
1,500 \\
240 \\
1,400 \\
1,000\end{array}$ & $\begin{array}{r}260 \\
180 \\
290 \\
270 \\
71\end{array}$ & $\begin{array}{r}125 \\
81 \\
180 \\
130 \\
29\end{array}$ & $\begin{array}{r}45 \\
40 \\
100 \\
55 \\
17\end{array}$ & $\begin{array}{r}16 \\
19 \\
43 \\
22 \\
9\end{array}$ & 21 & $\begin{array}{l}-610 \\
-470 \\
-370 \\
-260 \\
-140\end{array}$ \\
\hline $\begin{array}{l}\text { Be5T4 } \\
\text { 5BBe2 } \\
\text { 5BBe5 } \\
\text { 5BBe8 } \\
\text { BT5 }\end{array}$ & $\begin{array}{l}53.4 \\
48.3 \\
43.3 \\
42.1 \\
35.7\end{array}$ & $\begin{array}{l}50.5 \\
45.8 \\
42.0 \\
41.4 \\
35.4\end{array}$ & $\begin{array}{l}49.9 \\
45.1 \\
41.6 \\
41.2 \\
35.2\end{array}$ & $\begin{array}{l}49.2 \\
44.7 \\
41.3 \\
41.2 \\
35.0\end{array}$ & $\begin{array}{l}48.5 \\
44.2 \\
41.2 \\
41.1 \\
35.0\end{array}$ & $\begin{array}{l}48.1 \\
43.9 \\
41.0 \\
41.1 \\
35.0\end{array}$ & $\begin{array}{r}5,000 \\
2,600 \\
710 \\
430 \\
2,000\end{array}$ & $\begin{array}{r}820 \\
320 \\
280 \\
290 \\
1,400\end{array}$ & $\begin{array}{r}520 \\
160 \\
140 \\
170 \\
1,400\end{array}$ & $\begin{array}{r}290 \\
80 \\
63 \\
95 \\
1.100\end{array}$ & $\begin{array}{r}130 \\
40 \\
27 \\
46 \\
800\end{array}$ & $\begin{array}{r}63 \\
20 \\
15 \\
24 \\
500\end{array}$ & $\begin{array}{l}-590 \\
-540 \\
-310 \\
-130 \\
-120\end{array}$ \\
\hline $\begin{array}{l}\text { Be3T2... } \\
\text { 4BBe2 } \\
\text { 4BBe4... } \\
\text { 4BBe9.. } \\
\text { BT4 }\end{array}$ & $\begin{array}{l}43.8 \\
41.0 \\
41.3 \\
36.9 \\
35.6\end{array}$ & $\begin{array}{l}41.6 \\
39.1 \\
40.0 \\
36.2 \\
35.5\end{array}$ & $\begin{array}{l}41.0 \\
38.7 \\
39.6 \\
36.0 \\
35.5\end{array}$ & $\begin{array}{l}40.6 \\
38.3 \\
39.4 \\
36.1 \\
35.5\end{array}$ & $\begin{array}{l}40.3 \\
38.1 \\
39.3 \\
36.2 \\
35.6\end{array}$ & $\begin{array}{l}39.3 \\
36.5 \\
35.7\end{array}$ & $\begin{array}{r}3,000 \\
900 \\
2,000 \\
300 \\
3,000\end{array}$ & $\begin{array}{l}350 \\
230 \\
320 \\
400 \\
720\end{array}$ & $\begin{array}{r}170 \\
93 \\
130 \\
300 \\
330\end{array}$ & $\begin{array}{r}69 \\
42 \\
62 \\
200 \\
155\end{array}$ & $\begin{array}{l}34 \\
18 \\
28 \\
72 \\
78\end{array}$ & $\begin{array}{r}14 \\
35 \\
35\end{array}$ & $\begin{array}{r}-810 \\
-490 \\
-290 \\
-\end{array}$ \\
\hline $\begin{array}{l}\text { Be2T } \\
\text { 3BBel } \\
\text { 3B Be5 } \\
\text { 3B Be6 } \\
\text { 3B Be9 } \\
\text { BT3 }\end{array}$ & $\begin{array}{l}44.5 \\
46.3 \\
30.9 \\
31.0 \\
38.1 \\
44.5\end{array}$ & $\begin{array}{l}41.2 \\
43.8 \\
30.4 \\
30.9 \\
37.1 \\
43.8\end{array}$ & $\begin{array}{l}40.6 \\
43.2 \\
30.3 \\
30.9 \\
37.4 \\
43.6\end{array}$ & $\begin{array}{l}40.4 \\
42.5 \\
30.2 \\
30.8 \\
37.0 \\
43.5\end{array}$ & $\begin{array}{l}39.8 \\
42.4 \\
30.2 \\
30.8 \\
36.8 \\
43.2\end{array}$ & $\begin{array}{l}39.6 \\
42.2 \\
30.2 \\
30.8 \\
36.6 \\
42.9\end{array}$ & $\begin{array}{r}10,000 \\
800 \\
620 \\
260 \\
300 \\
700\end{array}$ & $\begin{array}{l}280 \\
330 \\
520 \\
330 \\
280 \\
600\end{array}$ & $\begin{array}{l}125 \\
130 \\
320 \\
290 \\
260 \\
600\end{array}$ & $\begin{array}{r}64 \\
54 \\
180 \\
210 \\
250 \\
600\end{array}$ & $\begin{array}{r}27 \\
24 \\
87 \\
140 \\
210 \\
600\end{array}$ & $\begin{array}{r}13 \\
12 \\
45 \\
83 \\
160 \\
600\end{array}$ & $\begin{array}{l}-610 \\
-550 \\
-130 \\
-35 \\
-230 \\
-210\end{array}$ \\
\hline $\begin{array}{l}\text { Be3T } \\
\text { 2BBel } \\
\text { 2BBe3 } \\
\text { 2BBe5 } \\
\text { 2BBe8 } \\
\text { 2BBe9 } \\
\text { BT2 }\end{array}$ & $\begin{array}{l}32.5 \\
29.1 \\
26.0 \\
22.6 \\
70.4 \\
163 \\
183\end{array}$ & $\begin{array}{l}31.3 \\
28.0 \\
26.0 \\
22.4 \\
73.8 \\
188 \\
222\end{array}$ & $\begin{array}{l}31.0 \\
27.6 \\
26.0 \\
22.4 \\
76.5 \\
212 \\
222\end{array}$ & $\begin{array}{l}30.6 \\
27.4 \\
26.1 \\
22.4 \\
75.9 \\
195 \\
174\end{array}$ & $\begin{array}{l}30.6 \\
27.3 \\
26.1 \\
22.4 \\
71.2 \\
160 \\
145\end{array}$ & $\begin{array}{l}30.8 \\
27.2 \\
26.15 \\
22.5 \\
66.4 \\
136 \\
125\end{array}$ & $\begin{array}{r}4,000 \\
170 \\
300 \\
170 \\
160 \\
62 \\
130\end{array}$ & $\begin{array}{r}300 \\
410 \\
110 \\
350 \\
210 \\
88 \\
220\end{array}$ & $\begin{array}{r}130 \\
210 \\
86 \\
300 \\
390 \\
170 \\
320\end{array}$ & $\begin{array}{r}50 \\
91 \\
59 \\
210 \\
800 \\
470 \\
440\end{array}$ & $\begin{array}{r}22 \\
41 \\
35 \\
110 \\
1,500 \\
1,000 \\
500\end{array}$ & $\begin{array}{r}12 \\
20 \\
22 \\
59 \\
1,400 \\
1,000 \\
600\end{array}$ & $\begin{array}{r}-380 \\
+50 \\
-(\mathrm{a})\end{array}$ \\
\hline $\begin{array}{l}\text { Be4T } \\
\text { 2BBe41 } \\
\text { 2B Be43 } \\
\text { 2B Be45 } \\
\text { 2B Be47 } \\
\text { 2B Be49 } \\
\text { B2T3 }\end{array}$ & $\begin{array}{l}22.9 \\
23.6 \\
20.5 \\
27.2 \\
161 \\
482 \\
796\end{array}$ & $\begin{array}{r}24.2 \\
23.1 \\
20.5 \\
27.3 \\
199 \\
586 \\
1,130\end{array}$ & $\begin{array}{l}24.6 \\
23.0 \\
20.5 \\
27.6 \\
264 \\
656 \\
961\end{array}$ & $\begin{array}{l}24.8 \\
23.0 \\
20.5 \\
27.6 \\
215 \\
550 \\
661\end{array}$ & $\begin{array}{c}24.9 \\
22.8 \\
20.6 \\
27.4 \\
163 \\
379 \\
487\end{array}$ & $\begin{array}{l}24.9 \\
22.8 \\
20.6 \\
27.2 \\
97 \\
286 \\
403\end{array}$ & $\begin{array}{r}51 \\
3,000 \\
83 \\
140 \\
44 \\
30 \\
60\end{array}$ & $\begin{array}{r}24 \\
490 \\
91 \\
130 \\
56 \\
59 \\
170\end{array}$ & $\begin{array}{r}29 \\
210 \\
96 \\
150 \\
110 \\
132 \\
360\end{array}$ & $\begin{array}{r}31 \\
93 \\
93 \\
140 \\
200 \\
320 \\
550\end{array}$ & $\begin{array}{r}27 \\
35 \\
79 \\
130 \\
190 \\
530 \\
700\end{array}$ & $\begin{array}{r}18 \\
22 \\
60 \\
110 \\
110 \\
640 \\
900\end{array}$ & $\begin{array}{r}+480 \\
-200 \\
+30 \\
\text { (a) }\end{array}$ \\
\hline $\begin{array}{l}\text { Be6T } \\
\text { BBe61 } \\
\text { BBe63 } \\
\text { BBe65 } \\
\text { BBe67 } \\
\text { BBe69 } \\
\text { BT }\end{array}$ & $\begin{array}{r}16.8 \\
16.4 \\
17.3 \\
64.0 \\
680 \\
1,190 \\
1,210\end{array}$ & $\begin{array}{l}17.0 \\
16.2 \\
17.3 \\
71.6 \\
906 \\
1,320 \\
1,850\end{array}$ & $\begin{array}{r}17.1 \\
16.1 \\
17.3 \\
76.1 \\
1,190 \\
4,140 \\
2,870\end{array}$ & $\begin{array}{r}17.2 \\
16.0 \\
17.2 \\
72.3 \\
900 \\
2,100 \\
1,550\end{array}$ & $\begin{array}{r}17.3 \\
16.0 \\
17.3 \\
62.5 \\
580 \\
1,250 \\
1,100\end{array}$ & $\begin{array}{l}17.4 \\
16.1 \\
17.3 \\
56.0 \\
428 \\
940 \\
820\end{array}$ & $\begin{array}{r}68 \\
150 \\
120 \\
78 \\
33 \\
75 \\
110\end{array}$ & $\begin{array}{r}49 \\
190 \\
110 \\
100 \\
56 \\
63 \\
47\end{array}$ & $\begin{array}{r}49 \\
160 \\
100 \\
160 \\
150 \\
67 \\
1,000\end{array}$ & $\begin{array}{r}44 \\
120 \\
99 \\
240 \\
380 \\
92 \\
1,000\end{array}$ & $\begin{array}{r}40 \\
68 \\
97 \\
210 \\
680 \\
67 \\
590\end{array}$ & $\begin{array}{r}19 \\
38 \\
81 \\
150 \\
730 \\
52 \\
190\end{array}$ & \begin{tabular}{r}
+210 \\
\hdashline \\
(a)
\end{tabular} \\
\hline
\end{tabular}

a Maximum $K$ value occurs between $100^{\circ}$ and $125^{\circ} \mathrm{C}$. 
TARLE 3. Dielectric constant, at $1 \mathrm{Mc} / \mathrm{s}$, from $-60^{\circ}$ to $+85^{\circ} \mathrm{C}$, and average temperature coefficient of dielectric constant

\begin{tabular}{|c|c|c|c|c|c|c|c|c|c|c|c|c|c|c|c|c|c|}
\hline \multirow{2}{*}{$\begin{array}{l}\text { Specimen } \\
\text { designation }\end{array}$} & \multicolumn{16}{|c|}{ Values of $K$ at ${ }^{\circ} \mathrm{C}-$} & \multirow{2}{*}{$\begin{array}{l}\text { Average } \\
\text { tempera- } \\
\text { ture coeffi- } \\
\text { cient of } K\end{array}$} \\
\hline & $-60^{\circ}$ & $-50^{\circ}$ & $-40^{\circ}$ & $-30^{\circ}$ & $-20^{\circ}$ & $-10^{\circ}$ & $0^{\circ}$ & $+10^{\circ}$ & $20^{\circ}$ & $30^{\circ}$ & $40^{\circ}$ & $50^{\circ}$ & $60^{\circ}$ & $70^{\circ}$ & $80^{\circ}$ & $85^{\circ}$ & \\
\hline $\begin{array}{l}\text { BeT3 } \\
\text { 18B Be6. } \\
\text { BT18 }\end{array}$ & $\begin{array}{l}84.8 \\
82.4 \\
80.5\end{array}$ & $\begin{array}{l}83.9 \\
81.6 \\
79.7\end{array}$ & $\begin{array}{l}82.9 \\
80.8 \\
78.9\end{array}$ & $\begin{array}{l}82.0 \\
80.1 \\
78.3\end{array}$ & $\begin{array}{l}81.2 \\
79.4 \\
77.6\end{array}$ & $\begin{array}{l}80.4 \\
78.8 \\
77.0\end{array}$ & $\begin{array}{l}79.8 \\
78.2 \\
76.5\end{array}$ & $\begin{array}{l}79.2 \\
77.6 \\
76.0\end{array}$ & $\begin{array}{l}78.8 \\
77.1 \\
75.4\end{array}$ & $\begin{array}{l}78.4 \\
76.6 \\
74.8\end{array}$ & $\begin{array}{l}77.7 \\
75.9 \\
74.3\end{array}$ & $\begin{array}{l}77.0 \\
75.4 \\
73.8\end{array}$ & $\begin{array}{l}76.4 \\
74.8 \\
73.4\end{array}$ & $\begin{array}{l}75.8 \\
74.2 \\
73.0\end{array}$ & $\begin{array}{l}75.3 \\
73.7 \\
72.6\end{array}$ & $\begin{array}{l}75.1 \\
73.4 \\
72.5\end{array}$ & $\begin{array}{r}p p m /{ }^{\circ} \mathrm{C} \\
-840 \\
-790 \\
-720\end{array}$ \\
\hline $\begin{array}{l}\mathrm{BeT} 2 \\
12 \mathrm{~B} \mathrm{Be} 4 \\
12 \mathrm{~B} \mathrm{Be} 7 \\
\mathrm{BT} 12\end{array}$ & $\begin{array}{l}75.9 \\
70.2 \\
70.4 \\
73.2\end{array}$ & $\begin{array}{l}75.0 \\
69.5 \\
69.8 \\
72.5\end{array}$ & $\begin{array}{l}74.2 \\
68.8 \\
69.1 \\
71.9\end{array}$ & $\begin{array}{l}73.4 \\
68.1 \\
68.7 \\
71.3\end{array}$ & $\begin{array}{l}72.7 \\
67.5 \\
68.1 \\
70.8\end{array}$ & $\begin{array}{l}72.1 \\
67.0 \\
67.7 \\
70.3\end{array}$ & $\begin{array}{l}71.5 \\
66.5 \\
67.2 \\
69.9\end{array}$ & $\begin{array}{l}71.0 \\
66.0 \\
66.8 \\
69.5\end{array}$ & $\begin{array}{l}70.4 \\
65.6 \\
66.5 \\
69.1\end{array}$ & $\begin{array}{l}69.9 \\
65.2 \\
66.1 \\
68.8\end{array}$ & $\begin{array}{l}69.4 \\
64.7 \\
65.7 \\
68.4\end{array}$ & $\begin{array}{l}68.8 \\
64.2 \\
65.3 \\
68.0\end{array}$ & $\begin{array}{l}68.2 \\
63.8 \\
64.8 \\
67.6\end{array}$ & $\begin{array}{l}67.7 \\
63.3 \\
64.5 \\
67.3\end{array}$ & $\begin{array}{l}67.2 \\
62.9 \\
64.1 \\
67.0\end{array}$ & $\begin{array}{l}67.0 \\
62.7 \\
63.9 \\
66.8\end{array}$ & $\begin{array}{l}-850 \\
-770 \\
-670 \\
-620\end{array}$ \\
\hline $\begin{array}{l}\text { Be2T3 } \\
\text { 9BBe3.- } \\
\text { 9B Be8 } \\
\text { BT9... }\end{array}$ & $\begin{array}{l}\text { 71.2 } \\
61.2 \\
64.2 \\
63.2\end{array}$ & $\begin{array}{l}70.5 \\
60.6 \\
63.6 \\
62.8\end{array}$ & $\begin{array}{l}69.7 \\
60.1 \\
63.1 \\
62.4\end{array}$ & $\begin{array}{l}69.0 \\
59.6 \\
62.7 \\
62.0\end{array}$ & $\begin{array}{l}68.4 \\
59.0 \\
62.4 \\
61.7\end{array}$ & $\begin{array}{l}67.8 \\
58.6 \\
62.0 \\
61.3\end{array}$ & $\begin{array}{l}67.2 \\
58.1 \\
61.7 \\
61.0\end{array}$ & $\begin{array}{l}66.7 \\
57.7 \\
61.3 \\
60.7\end{array}$ & $\begin{array}{l}66.1 \\
57.3 \\
61.0 \\
60.4\end{array}$ & $\begin{array}{l}65.6 \\
56.9 \\
60.6 \\
60.0\end{array}$ & $\begin{array}{l}65.1 \\
56.4 \\
60.3 \\
59.8\end{array}$ & $\begin{array}{l}64.6 \\
56.0 \\
60.0 \\
59.5\end{array}$ & $\begin{array}{l}64.1 \\
55.6 \\
59.7 \\
59.2\end{array}$ & $\begin{array}{l}63.7 \\
55.2 \\
59.4 \\
59.0\end{array}$ & $\begin{array}{l}63.2 \\
54.8 \\
59.1 \\
58.7\end{array}$ & $\begin{array}{l}63.0 \\
54.6 \\
58.9 \\
58.6\end{array}$ & $\begin{array}{l}-830 \\
-770 \\
-600 \\
-480\end{array}$ \\
\hline $\begin{array}{l}\text { BeT } \\
6 \mathrm{BBe} 2 \\
6 \mathrm{BBe} 5 \\
6 \mathrm{BBe} 8 \\
\text { BT6. }\end{array}$ & $\begin{array}{l}57.3 \\
53.7 \\
51.1 \\
49.2 \\
46.5\end{array}$ & $\begin{array}{l}56.7 \\
53.3 \\
50.8 \\
49.0 \\
46.3\end{array}$ & $\begin{array}{l}56.2 \\
52.9 \\
50.4 \\
48.8 \\
46.2\end{array}$ & $\begin{array}{l}55.7 \\
52.5 \\
50.1 \\
48.6 \\
46.0\end{array}$ & $\begin{array}{l}55.2 \\
52.1 \\
49.8 \\
48.4 \\
45.8\end{array}$ & $\begin{array}{l}54.7 \\
51.7 \\
49.5 \\
48.2 \\
45.6\end{array}$ & $\begin{array}{l}54.3 \\
51.4 \\
49.2 \\
48.1 \\
45.4\end{array}$ & $\begin{array}{l}53.9 \\
51.0 \\
49.0 \\
47.9 \\
45.3\end{array}$ & $\begin{array}{l}53.4 \\
50.6 \\
48.8 \\
47.8 \\
45.1\end{array}$ & $\begin{array}{l}53.0 \\
50.3 \\
48.5 \\
47.6 \\
45.0\end{array}$ & $\begin{array}{l}52.6 \\
49.9 \\
48.3 \\
47.5 \\
44.9\end{array}$ & $\begin{array}{l}52.2 \\
49.6 \\
48.0 \\
47.3 \\
44.8\end{array}$ & $\begin{array}{l}51.8 \\
49.3 \\
47.8 \\
47.1 \\
44.7\end{array}$ & $\begin{array}{l}51.5 \\
49.0 \\
47.6 \\
47.0 \\
44.6\end{array}$ & $\begin{array}{l}51.1 \\
48.7 \\
47.4 \\
46.9 \\
44.5\end{array}$ & $\begin{array}{l}50.9 \\
48.6 \\
47.3 \\
46.7 \\
44.5\end{array}$ & $\begin{array}{l}-800 \\
-700 \\
-520 \\
-360 \\
-300\end{array}$ \\
\hline $\begin{array}{l}\text { Be5T4 } \\
5 \mathrm{BBe2} \\
5 \mathrm{BBe} 5 \\
5 \mathrm{BBe} 8 \\
\text { BT5 }\end{array}$ & $\begin{array}{l}56.2 \\
50.8 \\
44.6 \\
42.4 \\
36.7\end{array}$ & $\begin{array}{l}55.5 \\
50.4 \\
44.3 \\
42.2 \\
36.7\end{array}$ & $\begin{array}{l}54.9 \\
50.0 \\
44.0 \\
42.1 \\
36.7\end{array}$ & $\begin{array}{l}54.4 \\
49.7 \\
43.8 \\
42.0 \\
36.7\end{array}$ & $\begin{array}{l}53.9 \\
49.3 \\
43.6 \\
41.9 \\
36.7\end{array}$ & $\begin{array}{l}53.5 \\
49.0 \\
43.4 \\
41.8 \\
36.7\end{array}$ & $\begin{array}{l}53.1 \\
48.6 \\
43.2 \\
41.7 \\
36.7\end{array}$ & $\begin{array}{l}52.8 \\
48.3 \\
43.0 \\
41.6 \\
36.7\end{array}$ & $\begin{array}{l}52.4 \\
48.0 \\
42.8 \\
41.6 \\
36.7\end{array}$ & $\begin{array}{l}52.0 \\
47.6 \\
42.6 \\
41.5 \\
36.7\end{array}$ & $\begin{array}{l}51.6 \\
47.3 \\
42.4 \\
41.4 \\
36.7\end{array}$ & $\begin{array}{l}51.2 \\
47.0 \\
42.2 \\
41.3 \\
36.7\end{array}$ & $\begin{array}{l}50.9 \\
46.7 \\
42.0 \\
41.3 \\
36.7\end{array}$ & $\begin{array}{l}50.5 \\
46.4 \\
41.9 \\
41.2 \\
36.7\end{array}$ & $\begin{array}{l}50.1 \\
46.1 \\
41.7 \\
41.1 \\
36.7\end{array}$ & $\begin{array}{l}50.0 \\
46.0 \\
41.6 \\
41.1 \\
36.7\end{array}$ & $\begin{array}{r}-800 \\
-680 \\
-870 \\
-220 \\
0\end{array}$ \\
\hline $\begin{array}{l}\mathrm{Be} 3 \mathrm{~T} 2 \\
4 \mathrm{BBe} 2 \\
4 \mathrm{BBe} 4 \\
4 \mathrm{BBe} 9 \\
\mathrm{BT} 4\end{array}$ & $\begin{array}{l}46.3 \\
42.9 \\
42.4 \\
35.5 \\
33.0\end{array}$ & $\begin{array}{l}45.9 \\
42.5 \\
42.2 \\
35.4 \\
33.1\end{array}$ & $\begin{array}{l}45.4 \\
42.1 \\
41.9 \\
35.4 \\
33.2\end{array}$ & $\begin{array}{l}45.0 \\
41.8 \\
41.7 \\
35.4 \\
33.3\end{array}$ & $\begin{array}{l}44.6 \\
41.5 \\
41.5 \\
35.4 \\
33.4\end{array}$ & \begin{tabular}{l|l}
44.2 & \\
41.2 & \\
41.3 & \\
35.3 & \\
33.5 &
\end{tabular} & $\begin{array}{l}43.9 \\
41.0 \\
41.1 \\
35.3 \\
33.6\end{array}$ & $\begin{array}{l}43.7 \\
40.7 \\
41.0 \\
35.3 \\
33.7\end{array}$ & $\begin{array}{l}43.4 \\
40.5 \\
40.8 \\
35.3 \\
33.7\end{array}$ & $\begin{array}{l}43.2 \\
40.2 \\
40.6 \\
35.3 \\
33.8\end{array}$ & $\begin{array}{l}42.9 \\
40.0 \\
40.4 \\
35.3 \\
33.9\end{array}$ & $\begin{array}{l}42.6 \\
39.7 \\
40.3 \\
35.3 \\
33.9\end{array}$ & $\begin{array}{l}42.3 \\
39.5 \\
40.1 \\
35.3 \\
34.0\end{array}$ & $\begin{array}{l}42.0 \\
39.2 \\
39.9 \\
35.3 \\
34.0\end{array}$ & $\begin{array}{l}41.7 \\
39.0 \\
39.8 \\
35.2 \\
34.0\end{array}$ & $\begin{array}{l}41.6 \\
38.9 \\
39.7 \\
35.2 \\
34.0\end{array}$ & $\begin{array}{r}-730 \\
-670 \\
-440 \\
-50 \\
+200\end{array}$ \\
\hline $\begin{array}{l}\text { Be2T } \\
\text { 3BBe1-... } \\
\text { 3BBe5- } \\
\text { 3B Be6-.. } \\
\text { 3BBe9.. } \\
\text { BT3 }\end{array}$ & $\begin{array}{l}43.4 \\
46.9 \\
31.1 \\
30.0 \\
36.8 \\
44.6\end{array}$ & $\begin{array}{l}43.0 \\
46.5 \\
31.0 \\
30.0 \\
36.8 \\
44.5\end{array}$ & $\begin{array}{l}42.6 \\
46.0 \\
30.9 \\
30.0 \\
36.8 \\
44.5\end{array}$ & $\begin{array}{l}42.2 \\
45.5 \\
30.8 \\
30.0 \\
36.8 \\
44.4\end{array}$ & $\begin{array}{l}41.9 \\
45.2 \\
30.7 \\
30.0 \\
36.7 \\
44.3\end{array}$ & $\begin{array}{l}41.6 \\
44.8 \\
30.7 \\
30.0 \\
36.7 \\
44.3\end{array}$ & $\begin{array}{l}41.3 \\
44.4 \\
30.6 \\
29.9 \\
36.7 \\
44.2\end{array}$ & $\begin{array}{l}41.1 \\
44.2 \\
30.6 \\
29.9 \\
36.7 \\
44.1\end{array}$ & $\begin{array}{l}40.8 \\
43.9 \\
30.5 \\
29.9 \\
36.7 \\
44.1\end{array}$ & $\begin{array}{l}40.5 \\
43.6 \\
30.4 \\
29.9 \\
36.7 \\
44.0\end{array}$ & $\begin{array}{l}40.2 \\
43.3 \\
30.4 \\
29.9 \\
36.7 \\
43.9\end{array}$ & $\begin{array}{l}40.0 \\
43.0 \\
30.3 \\
29.9 \\
36.7 \\
43.9\end{array}$ & $\begin{array}{l}39.7 \\
42.7 \\
30.3 \\
29.9 \\
36.6 \\
43.8\end{array}$ & $\begin{array}{l}39.4 \\
42.4 \\
30.2 \\
29.9 \\
36.6 \\
43.8\end{array}$ & $\begin{array}{l}39.2 \\
42.2 \\
30.2 \\
29.9 \\
36.6 \\
43.7\end{array}$ & $\begin{array}{l}39.1 \\
42.0 \\
30.2 \\
29.8 \\
36.6 \\
43.7\end{array}$ & $\begin{array}{r}-720 \\
-750 \\
-200 \\
-40 \\
-40 \\
-140\end{array}$ \\
\hline $\begin{array}{l}\text { Be3T } \\
2 \mathrm{BBe} 1 \\
2 \mathrm{BBe} 3 \\
2 \mathrm{BBe} 5 \\
2 \mathrm{BBe} 8 \\
\text { 2B Be9 } \\
\text { BT2 }\end{array}$ & $\begin{array}{l}30.5 \\
29.9 \\
24.6 \\
22.5 \\
63.2 \\
126 \\
163\end{array}$ & $\begin{array}{l}30.2 \\
29.7 \\
24.6 \\
22.5 \\
64.0 \\
128 \\
168\end{array}$ & $\begin{array}{l}30.0 \\
29.5 \\
24.5 \\
22.5 \\
64.8 \\
134 \\
173\end{array}$ & $\begin{array}{l}29.8 \\
29.3 \\
24.5 \\
22.5 \\
65.5 \\
138 \\
177\end{array}$ & \begin{tabular}{l|}
29.6 \\
29.1 \\
24.5 \\
22.6 \\
66.2 \\
142 \\
182
\end{tabular} & $\begin{array}{l}29.4 \\
28.9 \\
24.4 \\
22.6 \\
66.8 \\
146 \\
187\end{array}$ & $\begin{array}{l}29.3 \\
28.8 \\
24.4 \\
22.6 \\
67.5 \\
150 \\
192\end{array}$ & $\begin{array}{l}29.1 \\
28.7 \\
24.4 \\
22.6 \\
68.1 \\
153 \\
196\end{array}$ & $\begin{array}{l}29.0 \\
28.5 \\
24.4 \\
22.6 \\
68.7 \\
157 \\
200\end{array}$ & $\begin{array}{l}28.9 \\
28.4 \\
24.3 \\
22.6 \\
69.2 \\
159 \\
202\end{array}$ & $\begin{array}{c}28.7 \\
28.3 \\
24.3 \\
22.6 \\
69.6 \\
161 \\
204\end{array}$ & $\begin{array}{l}28.6 \\
28.1 \\
24.3 \\
22.6 \\
69.9 \\
163 \\
207\end{array}$ & $\begin{array}{l}28.5 \\
28.0 \\
24.3 \\
22.6 \\
70.1 \\
165 \\
210\end{array}$ & $\begin{array}{l}28.4 \\
27.9 \\
24.2 \\
22.6 \\
70.4 \\
167 \\
214\end{array}$ & $\begin{array}{l}28.3 \\
27.7 \\
24.2 \\
22.6 \\
70.9 \\
171 \\
219\end{array}$ & $\begin{array}{l}28.3 \\
27.7 \\
24.2 \\
22.6 \\
71.3 \\
173 \\
221\end{array}$ & $\begin{array}{r}-510 \\
-510 \\
-120 \\
+20 \\
+820 \\
+2,180 \\
+2,080\end{array}$ \\
\hline $\begin{array}{l}\mathrm{Be} 4 \mathrm{~T} \\
\text { 2BBe41 } \\
\text { 2B Be43 } \\
\text { 2B Be45 } \\
\text { 2B Be47 } \\
\text { 2B Be49 } \\
\text { B2T3 }\end{array}$ & $\begin{array}{r}24.5 \\
23.8 \\
20.5 \\
26.0 \\
120 \\
311 \\
600\end{array}$ & $\begin{array}{l}24.3 \\
23.7 \\
20.5 \\
26.1 \\
125 \\
330 \\
630\end{array}$ & $\begin{array}{l}24.2 \\
23.6 \\
20.5 \\
26.2 \\
132 \\
350 \\
660\end{array}$ & $\begin{array}{l}24.0 \\
23.5 \\
20.5 \\
26.3 \\
137 \\
365 \\
690\end{array}$ & $\begin{array}{c}23.9 \\
23.4 \\
20.5 \\
26.3 \\
143 \\
385 \\
720\end{array}$ & $\begin{array}{c}23.8 \\
23.4 \\
20.5 \\
26.4 \\
148 \\
405 \\
745\end{array}$ & $\begin{array}{l}23.7 \\
23.3 \\
20.5 \\
26.5 \\
154 \\
430 \\
780\end{array}$ & $\begin{array}{l}23.6 \\
23.2 \\
20.5 \\
26.5 \\
159 \\
450 \\
825\end{array}$ & $\begin{array}{l}23.6 \\
23.2 \\
20.5 \\
26.6 \\
164 \\
470 \\
870\end{array}$ & $\begin{array}{c}23.5 \\
23.1 \\
20.6 \\
26.6 \\
167 \\
480 \\
885\end{array}$ & $\begin{array}{c}23.4 \\
23.0 \\
20.6 \\
26.7 \\
170 \\
490 \\
900\end{array}$ & $\begin{array}{c}23.4 \\
23.0 \\
20.6 \\
26.7 \\
173 \\
500 \\
910\end{array}$ & $\begin{array}{c}23.3 \\
22.9 \\
20.6 \\
26.8 \\
177 \\
510 \\
920\end{array}$ & $\begin{array}{l}23.3 \\
22.9 \\
20.6 \\
26.8 \\
182 \\
520 \\
935\end{array}$ & $\begin{array}{l}23.4 \\
22.8 \\
20.6 \\
26.8 \\
187 \\
535 \\
960\end{array}$ & $\begin{array}{l}23.4 \\
22.8 \\
20.6 \\
26.9 \\
191 \\
543 \\
980\end{array}$ & $\begin{array}{r}-310 \\
+50 \\
+220 \\
+3,150 \\
+3,750 \\
+3,300\end{array}$ \\
\hline $\begin{array}{l}\text { Be6T } \\
\text { BBe61 } \\
\text { BBe63- } \\
\text { BBe65 } \\
\text { BBe67 } \\
\text { BBe69 } \\
\text { BT }\end{array}$ & $\begin{array}{l}16.5 \\
16.5 \\
16.7 \\
53.8 \\
419 \\
638 \\
805\end{array}$ & $\begin{array}{c}16.5 \\
16.5 \\
16.7 \\
55.1 \\
450 \\
660 \\
815\end{array}$ & $\begin{array}{l}16.4 \\
16.5 \\
16.7 \\
56.5 \\
480 \\
680 \\
860\end{array}$ & $\begin{array}{l}16.4 \\
16.4 \\
16.7 \\
57.8 \\
510 \\
710 \\
910\end{array}$ & $\begin{array}{l}16.4 \\
16.4 \\
16.7 \\
59.0 \\
540 \\
740 \\
980\end{array}$ & $\begin{array}{c}16.3 \\
16.4 \\
16.8 \\
60.1 \\
570 \\
780 \\
1,060\end{array}$ & $\begin{array}{c}16.3 \\
16.3 \\
16.8 \\
61.2 \\
600 \\
830 \\
1,160\end{array}$ & $\begin{array}{c}16.3 \\
16.3 \\
16.8 \\
62.1 \\
630 \\
910 \\
1,310\end{array}$ & $\begin{array}{c}16.2 \\
16.3 \\
16.8 \\
63.2 \\
660 \\
1,140 \\
1,570\end{array}$ & $\begin{array}{c}16.2 \\
16.3 \\
16.8 \\
64.1 \\
680 \\
1,105 \\
1,420\end{array}$ & $\begin{array}{r}16.2 \\
16.2 \\
16.8 \\
64.8 \\
705 \\
1,055 \\
1,360\end{array}$ & $\begin{array}{c}16.2 \\
16.2 \\
16.8 \\
65.4 \\
710 \\
1,015 \\
1,330\end{array}$ & $\begin{array}{c}16.2 \\
16.2 \\
16.8 \\
66.0 \\
730 \\
1,005 \\
1,300\end{array}$ & $\begin{array}{c}16.2 \\
16.2 \\
16.8 \\
66.6 \\
744 \\
1,000 \\
1,330\end{array}$ & $\begin{array}{r}16.2 \\
16.2 \\
16.8 \\
67.4 \\
775 \\
1,030 \\
1,400\end{array}$ & $\begin{array}{r}16.2 \\
16.2 \\
16.8 \\
67.9 \\
790 \\
1,048 \\
1,440\end{array}$ & $\begin{array}{r}-120 \\
-160 \\
+40 \\
+1,600 \\
+4,260 \\
\end{array}$ \\
\hline
\end{tabular}




\begin{tabular}{|c|c|c|c|c|c|c|c|c|}
\hline \multirow{2}{*}{$\begin{array}{l}\text { Specimen } \\
\text { designation }\end{array}$} & \multicolumn{4}{|c|}{ Dielectric constant, $K$} & \multicolumn{4}{|c|}{ Quality factor, $Q$} \\
\hline & $\begin{array}{c}\text { After } 1 \\
\text { day }\end{array}$ & $\begin{array}{l}\text { After } 6 \\
\text { months }\end{array}$ & Change & $\begin{array}{l}\text { A versge } \\
\text { change }\end{array}$ & $\begin{array}{l}\text { After } 1 \\
\text { day }\end{array}$ & $\begin{array}{l}\text { After } 6 \\
\text { months }\end{array}$ & Change & $\begin{array}{l}\text { Average } \\
\text { change }\end{array}$ \\
\hline \multicolumn{9}{|c|}{ Specimens with significant change in $K$} \\
\hline $\begin{array}{r}2 \mathrm{BBe} 9-2 \ldots \\
-3 . \\
-4\end{array}$ & $\begin{array}{l}161 \\
156 \\
152\end{array}$ & $\begin{array}{l}157 \\
154 \\
150\end{array}$ & $\begin{array}{c}\text { Percent } \\
-2 \\
-1 \\
-1\end{array}$ & Percent & $\begin{array}{l}49 \\
55 \\
62\end{array}$ & $\begin{array}{l}51 \\
53 \\
62\end{array}$ & $\begin{array}{c}\text { Percent } \\
+4 \\
-4 \\
0\end{array}$ & $\begin{array}{c}\text { Percent } \\
0 \\
0\end{array}$ \\
\hline $\begin{array}{r}2 \mathrm{BBe} 47 \\
-1 \\
-\mathrm{B}\end{array}$ & $\begin{array}{l}167 \\
190 \\
177\end{array}$ & $\begin{array}{l}165 \\
186 \\
173\end{array}$ & $\begin{array}{l}-1 \\
-2 \\
-2\end{array}$ & -2 & $\begin{array}{l}32 \\
28 \\
36\end{array}$ & $\begin{array}{l}19 \\
29 \\
38\end{array}$ & $\begin{array}{r}-40 \\
+4 \\
+6\end{array}$ & $-10 ?$ \\
\hline $\begin{array}{r}2 \mathrm{BBe} 49 \\
-3 . \\
-4\end{array}$ & $\begin{array}{l}479 \\
462 \\
454\end{array}$ & $\begin{array}{l}463 \\
450 \\
444\end{array}$ & $\begin{array}{l}-3 \\
-3 \\
-2\end{array}$ & -3 & $\begin{array}{l}30 \\
31 \\
33\end{array}$ & $\begin{array}{l}30 \\
31 \\
33\end{array}$ & $\begin{array}{l}0 \\
0 \\
0\end{array}$ & 0 \\
\hline B Be67 & $\begin{array}{l}692 \\
665 \\
671\end{array}$ & $\begin{array}{l}654 \\
645 \\
668\end{array}$ & $\begin{array}{r}-4 \\
-3 \\
-1,2\end{array}$ & $-21 / 2$ & $\begin{array}{l}27 \\
29 \\
27\end{array}$ & $\begin{array}{l}24 \\
29 \\
24\end{array}$ & $\begin{array}{r}-11 \\
0 \\
-11\end{array}$ & $-4 ?$ \\
\hline $\begin{array}{r}\text { BBe69-2.... } \\
-4 \\
-5 \\
-8 \\
\end{array}$ & $\begin{array}{l}1,110 \\
1.120 \\
1,097 \\
1,140\end{array}$ & $\begin{array}{l}1,080 \\
1,096 \\
1,060 \\
1,074\end{array}$ & $\begin{array}{l}-3 \\
-2 \\
-3 \\
-6\end{array}$ & $-31 / 2$ & $\begin{array}{l}39 \\
58 \\
60 \\
53\end{array}$ & $\begin{array}{l}40 \\
64 \\
66 \\
55\end{array}$ & $\begin{array}{r}+2 \\
+10 \\
+10 \\
+4\end{array}$ & +7 \\
\hline \multicolumn{9}{|c|}{ Specimens with significant change in $Q$} \\
\hline $\begin{array}{r}3 \mathrm{BBe} 5 \ldots \\
-1\end{array}$ & $\begin{array}{l}30.5 \\
30.5\end{array}$ & $\begin{array}{l}30.6 \\
30.1\end{array}$ & - & (n) & $\begin{array}{r}830 \\
1,600\end{array}$ & $\begin{array}{l}180 \\
600\end{array}$ & $\begin{array}{l}-78 \\
-63\end{array}$ & -70 \\
\hline $3 \mathrm{BBe} 6-1 \ldots$ & $\begin{array}{l}28.4 \\
28.9\end{array}$ & $\begin{array}{r}\text { a } 29.5 \\
29.2\end{array}$ & - & . & $\begin{array}{r}1,000 \\
430\end{array}$ & $\begin{array}{l}31 \\
55\end{array}$ & $\begin{array}{l}-97 \\
-87\end{array}$ & -92 \\
\hline $\begin{array}{r}3 \mathrm{BBe} 9 \\
-\mathrm{A} \\
-\mathrm{B} \\
\end{array}$ & $\begin{array}{l}37.4 \\
36.7 \\
36.9\end{array}$ & $\begin{array}{r}36.8 \\
36.9 \\
37.2\end{array}$ & - & - & $\begin{array}{r}1,100 \\
200 \\
140\end{array}$ & $\begin{array}{r}470 \\
91 \\
51\end{array}$ & $\begin{array}{l}-57 \\
-55 \\
-64\end{array}$ & -59 \\
\hline $2 \mathrm{BBe} 3 \ldots$ & $\begin{array}{l}24.6 \\
24.4\end{array}$ & $\begin{array}{r}\text { a } 27.0 \\
24.4\end{array}$ & - & - & $\begin{array}{l}330 \\
160\end{array}$ & $\begin{array}{r}8 \\
92\end{array}$ & $\begin{array}{l}-98 \\
-43\end{array}$ & -70 \\
\hline $\begin{array}{r}2 \mathrm{BBe} 5-1 \\
-\mathrm{A} \\
-\mathrm{C} \\
\end{array}$ & $\begin{array}{l}25.0 \\
22.0 \\
22.6\end{array}$ & $\begin{array}{l}25.3 \\
22.0 \\
22.7\end{array}$ & - & - & $\begin{array}{r}1,000 \\
400 \\
400\end{array}$ & $\begin{array}{l}800 \\
260 \\
330\end{array}$ & $\begin{array}{l}-20 \\
-35 \\
-18\end{array}$ & -24 \\
\hline BBe43-1.... & $\begin{array}{l}18.6 \\
20.1\end{array}$ & $\begin{array}{r}\text { a } 19.3 \\
20.3\end{array}$ & - & - & $\begin{array}{l}600 \\
150\end{array}$ & $\begin{array}{l}28 \\
26\end{array}$ & $\begin{array}{l}-95 \\
-83\end{array}$ & -90 \\
\hline $\mathrm{BBe} 45$ & $\begin{array}{l}26.0 \\
26.5\end{array}$ & $\begin{array}{l}26.0 \\
26.7\end{array}$ & - & - & $\begin{array}{l}270 \\
180\end{array}$ & $\begin{array}{r}190 \\
62\end{array}$ & $\begin{array}{l}-30 \\
-66\end{array}$ & -48 \\
\hline $\begin{array}{r}\mathrm{BBe} 65 \\
-\mathrm{A} \\
-\mathrm{B}\end{array}$ & $\begin{array}{l}63.0 \\
62.6 \\
62.6\end{array}$ & $\begin{array}{l}63.2 \\
62.4 \\
62.3\end{array}$ & - & - & $\begin{array}{l}68 \\
58 \\
59\end{array}$ & $\begin{array}{l}26 \\
52 \\
55\end{array}$ & $\begin{array}{r}-62 \\
-10 \\
-7\end{array}$ & -26 \\
\hline
\end{tabular}

a Apparent change not significant due to small capacitance of specimen.

\begin{tabular}{|c|c|c|c|c|c|c|c|}
\hline \multirow{2}{*}{ Specimen designation } & \multicolumn{7}{|c|}{ Temperature range from $25^{\circ} \mathrm{C}$ to -} \\
\hline & $100^{\circ}$ & $200^{\circ}$ & $300^{\circ}$ & $400^{\circ}$ & $500^{\circ}$ & $600^{\circ}$ & $700^{\circ}$ \\
\hline $\begin{array}{l}\text { 6BeO: } \mathrm{TiO}_{2} \\
\text { 4BeO:TiO } \\
\text { 2BeO:TiO } \\
\text { BeO:TiO } \\
\text { 5BBe5 } \\
\text { BBe65 }\end{array}$ & $\begin{array}{c}\text { Percent } \\
0.04 \\
.04 \\
.05 \\
.04 \\
.05 \\
.05\end{array}$ & $\begin{array}{c}\text { Percent } \\
0.11 \\
.12 \\
.12 \\
.11 \\
.14 \\
.13\end{array}$ & $\begin{array}{c}\text { Percent } \\
0.19 \\
.20 \\
.21 \\
.21 \\
.24 \\
.22\end{array}$ & $\begin{array}{c}\text { Percent } \\
0.28 \\
.33 \\
.30 \\
.33 \\
.33 \\
.33\end{array}$ & $\begin{array}{c}\text { Percent } \\
0.38 \\
.46 \\
.39 \\
.49 \\
.43 \\
.43\end{array}$ & $\begin{array}{c}\text { Percent } \\
0.48 \\
.58 \\
.49 \\
.68 \\
.53 \\
.54\end{array}$ & $\begin{array}{c}\text { Percent } \\
0.58 \\
.69 \\
.59 \\
.77 \\
.64 \\
.65\end{array}$ \\
\hline
\end{tabular}

TABLE 4.

Changes in $K$ and $Q$ of specimens after 6 months storage

(Measured at $1 \mathrm{Mc} / \mathrm{s}$ and $25^{\circ} \mathrm{C}$ )

\begin{tabular}{|c|c|c|c|c|c|}
\hline \multirow{2}{*}{$\begin{array}{l}\text { Specimen desig- } \\
\text { nation }\end{array}$} & \multicolumn{5}{|c|}{ Resistivity in ohm-cm after- } \\
\hline & $1 \mathrm{hr}$ & 1 day & 2 days & 5 days & 21 days \\
\hline $\begin{array}{l}\mathrm{BaTiO}_{3} \\
\mathrm{TiO}_{2} \\
5 \mathrm{BBe} 2 \\
2 \mathrm{BBe} 5 \\
\mathrm{BBe} 61 \\
\mathrm{BBe} 67\end{array}$ & $\begin{array}{l}6 \times 10^{6} \\
>10^{10} \\
>10^{10} \\
>10^{10} \\
>10^{10} \\
>10^{10}\end{array}$ & $\begin{aligned} & 8 \times 10^{5} \\
& 10^{9} \\
> & 10^{10} \\
> & 10^{10} \\
> & 10^{10} \\
> & 10^{10}\end{aligned}$ & $\begin{array}{r}a 8 \times 10^{5} \\
2 \times 10^{8} \\
4 \times 10^{9} \\
>10^{10} \\
>10^{10} \\
>10^{10}\end{array}$ & $\begin{aligned} & 10^{6} \\
& 10^{5} \\
> & 10^{10} \\
> & 10^{10} \\
> & 10^{10}\end{aligned}$ & $\begin{array}{r}{ }^{\mathrm{b}} 6 \times 10^{5} \\
{ }^{\mathrm{e}} 5 \times 10^{6} \\
>10^{10} \\
>10^{10} \\
>10^{10}\end{array}$ \\
\hline
\end{tabular}

TABle 6. Approximate volume resistivity of some specimens, 0.010 in thick, measured at $200^{\circ} \mathrm{C}$ and at a potential of 30-v direct current maintained for various periods of time

a In $2 \mathrm{hr}$ after 2 days, the resistivity began to decreasegrapidly and, within an additional $2 \mathrm{hr}$ had decreased to $1 \times 10^{5} \mathrm{ohm}-\mathrm{cm}$, when test was discontinued.

b After 7 days.

c After 15 days.

Washington, December 19, 1950. 